\title{
Response to: Rethinking Human Development and the Shared Intentionality Hypothesis
}

\section{Michael Tomasello ${ }^{1,2}$}

Published online: 15 September 2020

(C) The Author(s) 2020

\begin{abstract}
I respond to Moll, Nichols, and Mackey's review of my book Becoming Human. I agree with many of their points, but have my own point of view on some others.
\end{abstract}

It is extremely rare for me to read a review of one of my books that really gets it. I would therefore like to thank Moll, Nichols, and Mackey (MNM) for taking the time and putting in the effort to really understand Becoming Human; their review advances the argument in highly productive ways. I have two very small nits to pick, which I will do at the end, but, in general, the summarizing parts of the review are insightful and in all ways excellent, even highlighting for me some changes of theory I hadn't even realized I had made.

MNM proffer three important critiques. The first is the way that I applied the term second personal to children's development. I based my account mainly on the work of Stephen Darwall (e.g., 2006) and, as a moral philosopher, he has a very demanding definition: second-personal agents are persons who hold one another accountable and so respect and are responsible to one another. I argue that children only become fully second-personal agents in this sense at 3 years of age, when they begin treating one another with a sense of respect and fairness and calling one another out for transgressions. But MNM rightly point out that in a more expansive concept of second personal agency, toddlers prior to 3 years of age qualify: they interact with others in joint attention, they address one another communicatively (second personally), and, in general, interact with others as intentional agents with whom one may share intentional states. My nod in this direction was to say, as MNM quote, that while 2-year-olds are not yet fully second-personal agents, "they are working on it": they have some parts of the second-personal stance, as it is sometimes called, but perhaps not all parts.

One fact that MNM do not highlight is relevant here. One- and two-year-old toddlers are engaging in all of these second-personal ways almost exclusively with adults, not

Michael Tomasello

michael.tomasello@duke.edu

1 Department of Psychology and Neuroscience, Duke University, Durham, NC 27701, USA

2 Max Planck Institute for Evolutionary Anthropology, Leipzig, Germany 
with peers (a point that I emphasize in various parts of the book). If toddlers interacted with one another in all of these unique and interesting ways, this would signal a deeper (more adult-like) appreciation for this mode of interaction, since it would involve them applying it not to an all-powerful and all-knowing adult, but to a coequal peer. So let us compromise. I have no problem replacing my account that "they are working on it" with something a bit richer and more specific, for example, an account which says that 1- and 2-year-old toddlers are engaging with adults as second-personal agents when they participate with them in joint attention and cooperative communication. But then the capstone of this developmental process occurs at age 3, when children start to treat partners, including peers, normatively with respect and fairness as equals, and to demand such treatment from partners in return.

The second issue is the way that I have characterized 3-year-olds' group-mindedness. Here I confess to a somewhat confusing (and perhaps confused) account. But at the same time I have trouble coming up with a viable alternative, and I do not believe that MNM have provided one either. The issue is how to understand the "normative turn" at around 3 years of age, as best exemplified in children's tendency to enforce social norms on others, often using the normative language of should, must, and ought. I attempted to characterize this understanding by hypothesizing that children understood social norms as coming from the cultural group, with the norm enforcer as individual representative. MNM are right to call me out on this: it is unlikely that 3year-olds think in this way explicitly. By characterizing things in the way that I did, I was kind of sneaking the evolutionary bases of normative understanding - as based in group-minded thinking - into children's understanding, which is more likely based on something simpler. But MNM's alternative account is too normatively "thin". They propose that toddlers trust adults based on their familiarity with them, without thinking in terms of group at all, and that children presuppose that what adults teach is objective without conceptualizing it as coming from the culture. In their view, the 'oughts' with which children begin to work at this age are seen as just more furniture in the objective world.

But if normative standards are pieces of furniture in the world, they are very special pieces of furniture - and they must be understood as special to carry the force that they do. Social norms represent another mode of existence. As philosophers throughout recorded history have recognized, the normative world is not the actual world but rather a possible world, that is, a possible world that there is good reason to bring about. Evolutionarily, I believe that the only way this way of thinking could have come into existence is by individuals recognizing that the cultural group with which they identify values the realization of certain possible worlds in certain situations. The identification part is crucial, as this gets us past the cultural group as just a bunch of individuals to some kind of ideal based on "what anyone who would be one of us" ideally should think or do in the situation. To break with these ideals is thus to lose my cultural identity, and this supplies the strong force of norms. Evolutionarily, I have argued that this normally takes place in contrast to other groups. But the earliest modern humans thought that other groups were not human but "barbarian", and so they were not opposing one human way of thinking about things with another, but simply characterizing the right human way to think about things (since "we" in our group are the only human beings). So I do not think that young children have to think about an opposing 
group to think normatively; they only need to think about how "we" think about and do things.

This having been said, of course young children are not thinking like this consciously. But neither do I think that they are thinking of norms as just more pieces of world furniture. We all may think this way when things are going smoothly, but when someone, including ourselves, breaks a norm, that conceptualization no longer works: the actual world and a valued possible world collide, and we know which one ought to come out on top. I do not know exactly the best way to characterize things here; but I have good company in my ignorance. One way or the other, discovering how children understand and conceptualize cultural norms is a pressing issue for future theoretical work in developmental psychology.

The third issue concerns my attempt to characterize the objective/normative turn at 3 years of age as including children's understanding of multi-perspectival concepts, including false belief. Part of the difficulty is a misunderstanding that I engendered by speaking too loosely. I did not intend to posit a genuine $U$-shaped developmental trajectory in children's understanding of false belief (i.e., success, followed by failure, followed by a return to success). MNM point out my loose talk about this. What I intended to say was that children's understanding does indeed, as MNM rightly claim, progress across the preschool period. There is an apparent U-shaped development because they pass infant false belief tasks, then fail so-called explicit tasks, and then pass so-called explicit tasks. But the infant and explicit tasks are measuring different things, as MNM point out, and so I can only apologize for my sloppy talk and stand corrected.

However, another part of their argument is that young children only come to understand false belief at 4 to 5 years of age, and they characterize my attempt to include the understanding of beliefs as part of the objective/normative turn at 3 years of age as an attempt to fit the data into my Procrustean theoretical bed. But this is an issue that should have an empirical resolution. Here is what I would claim. Children have the ability to understand false beliefs from soon after 3 years of age, and indeed there are a number of studies in which researchers lower the task demands involved and then 3-year-olds begin to pass the task (I cite some of these in Chapter 3). So why do 3-year-olds fail the standard task? This is where I invoked the research of Moll herself (e.g., Moll et al. 2013; Moll and Tomasello 2012). Three-year-olds struggle similarly (and 4 to 5-year-olds pass) in tasks involving other multi-perspectival concepts, such as visual perspective-taking, appearance-reality, and dual naming. The difficulty in all of these cases, I argue, is coordinating the different perspectives involved in a way leading to a satisfactory resolution: an object appears differently to you and me because we are viewing it from different viewing angles; the object appears to be a rock but it's really a sponge depending on how one inspects it perceptually; and one and the same object is a cow and an animal because words can be conventionalized at different taxonomic levels. The point is that constructing these concepts requires something more than just an objective/normative way of looking at things; it requires the skills of executive control necessary to coordinate multiple perspectives into one conceptualization. It is well-known that executive function is developing rapidly during exactly this age range, and it is well-known that skills of executive function correlate very strongly with young children's false belief understanding. So MNM's characterization of children's performance on these tasks across the preschool period is correct, but the underlying explanation, in my view, does not involve just the ability to take an objective perspective, but also executive skills for coordinating different perspectives in 
relation to an objective perspective. (By the way, do we really know that 2-year-olds make the realist error in the false belief task? I know of no good data, and MNM provide no citations for their claim that they do.)

Finally, the two nits I promised to pick. First, MNM claim that I explicitly endorse the recapitulation thesis that ontogeny recapitulates phylogeny. They may not have meant it in this way, but this implies that I believe it must recapitulate it. But this is clearly not my view, as all of my other writings demonstrate. What I claimed in the quote they recite is that in this particular case - that is, the transition from joint intentionality to collective intentionality - ontogeny does indeed follow the same ordering as phylogeny (which might even be surprising). Second, MNM claim that I am inconsistent in my application of the transformative account of how the transition from great ape individual intentionality to human shared intentionality takes place - because in some places I talk about infants and apes doing "basically the same thing" and that shared intentionality is an "extra something" that distinguishes humans from apes. Perhaps my wording here was imprecise, but I would say now as clearly as I can that when viewing human ontogeny as a whole these accounts are not mutually exclusive. It is easy to imagine that some things are additive and others are transformative. I do not believe that color perception or object recognition or the emotion of fear are transformed by the transition from apes to humans, only some aspects of their cognition and sociality. In any case, I do not subscribe to the thesis that a transformative account - to which I subscribe in general - must of necessity apply to every single aspect of human psychology without exception.

Again I would like to thank Moll, Nichols, and Mackey for their very thoughtful and insightful review, and I would like to thank the editors for giving me the opportunity to respond to their stimulating questions and critiques. I hope the dialogue can serve to move the field forward on these very important issues.

Funding Open Access funding enabled and organized by Projekt DEAL.

Open Access This article is licensed under a Creative Commons Attribution 4.0 International License, which permits use, sharing, adaptation, distribution and reproduction in any medium or format, as long as you give appropriate credit to the original author(s) and the source, provide a link to the Creative Commons licence, and indicate if changes were made. The images or other third party material in this article are included in the article's Creative Commons licence, unless indicated otherwise in a credit line to the material. If material is not included in the article's Creative Commons licence and your intended use is not permitted by statutory regulation or exceeds the permitted use, you will need to obtain permission directly from the copyright holder. To view a copy of this licence, visit http://creativecommons.org/licenses/by/4.0/.

\section{References}

Darwall, S. 2006. The second-person standpoint: Respect, morality, and accountability. Cambridge: Harvard University Press.

Moll, H., A.N. Meltzoff, K. Merzsch, and M. Tomasello. 2013. Taking versus confronting visual perspectives in preschool children. Developmental Psychology 49 (4): 646-654.

Moll, H., and M. Tomasello. 2012. Three-year-olds understand appearance and reality - just not about the same object at the same time. Developmental Psychology 48 (4): 1124-1132.

Publisher's Note Springer Nature remains neutral with regard to jurisdictional claims in published maps and institutional affiliations. 